OPEN ACCESS

Edited by:

Mario J. P. F. G. Monteiro, Universidade do Porto, Portugal

Reviewed by:

João Manuel Fernandes, University of Coimbra, Portugal Joyce Ann Guzik, Los Alamos National Laboratory

(DOE), United States

*Correspondence:

Scilla Degl'Innocenti scilla.deglinnocenti@unipi.it

Specialty section:

This article was submitted to Stellar and Solar Physics,

a section of the journal

Frontiers in Astronomy and Space

Sciences

Received: 11 February 2019 Accepted: 08 April 2019 Published: 26 April 2019

Citation:

Valle $G$, Dell'Omodarme M,

Prada Moroni PG and Degl'Innocenti S (2019) Mixing-Length Estimates From Binary Systems. A Theoretical Investigation on the Estimation Errors.

Front. Astron. Space Sci. 6:30. doi: 10.3389/fspas.2019.00030

\section{Mixing-Length Estimates From Binary Systems. A Theoretical Investigation on the Estimation Errors}

\author{
Giada Valle ${ }^{1,2}$, Matteo Dell'Omodarme ${ }^{1}$, Pier G. Prada Moroni ${ }^{1,2}$ and \\ Scilla Degl'Innocenti ${ }^{1,2 *}$ \\ 'Dipartimento di Fisica "Enrico Fermi," Università di Pisa, Pisa, Italy, ${ }^{2}$ Istituto Nazionale di Fisica Nucleare (INFN), Sezione di \\ Pisa, Pisa, Italy
}

We performed a theoretical investigation on the biases and random uncertainties affecting the recovery of the mixing-length parameter $\alpha_{\mathrm{ml}}$ from an ideal eclipsing double-lined binary system, with well constrained masses and radii. We focused on a test case composed by a primary of mass $M=0.95 M_{\odot}$ and a secondary of $M=0.85 M_{\odot}$. Synthetic stars were generated coevally and with a common chemical composition by sampling from a dense grid of stellar models. Observational errors were simulated by adding random perturbations to mock data. The $\alpha_{\mathrm{ml}}$ parameter was then recovered by means of the SCEPtER-binary pipeline. Several Monte Carlo simulations were conducted considering three metallicities, coupled to three different evolutionary stages of the primary. For each configuration, artificial data were sampled assuming an increasing difference between the mixing-length of the two stars. The mixing length values were then reconstructed adopting three alternative set-ups. A first method, which assumes full independence between the two stars, showed a great difficulty to constrain the mixing-length values; the recovered values were nearly unconstrained with a standard deviation of about 0.40 . The second technique imposes the constraint of common age and initial chemical composition for the two stars in the fit. We found that $\alpha_{\mathrm{ml}, 1}$ values closely match the ones recovered under the previous configuration, but $\alpha_{\mathrm{ml}, 2}$ values are much more peaked around unbiased estimates. This occurs because the primary star provides a much tighter age constraint in the joint fit than the secondary, thus leading to the rejection of several extreme solutions for the secondary. Within this second scenario we also explored, for systems sharing a common $\alpha_{\mathrm{ml}}=2.0$, the difference in the mixing-length values of the two stars only due to random fluctuations owing to the observational errors. The posterior distribution of these differences was peaked around zero, with a somewhat large standard deviation of 0.3 (about $15 \%$ of the solar-scaled value). Therefore, about $32 \%$ of systems with true identical $\alpha_{\mathrm{ml}}$ are expected to show differences higher than that only owing to random errors. The third technique also imposes the constraint of a common mixing-length value for the two stars. This assumption is generally not true for the sample stars and served as a test for identifying wrong fitting assumptions. In this 
case, the common mixing-length is mainly dictated by the value of $\alpha_{\mathrm{ml}, 2}$. However, an increasing share of systems cannot be fitted by the algorithm as the differences of $\alpha_{\mathrm{ml}}$ between the two stars in the synthetic systems increases. For $\Delta \alpha_{\mathrm{ml}}>0.4$, less than half of the systems can be recovered and only $20 \%$ at $\Delta \alpha_{\mathrm{ml}}=1.0$.

Keywords: mixing-length parameter, eclipsing binaries, stellar evolution, statistical analysis, Monte Carlo simulations

\section{INTRODUCTION}

Despite the substantial refinements of the accuracy and reliability of stellar evolutionary predictions, several mechanisms involved in the evolution of stars are still poorly understood. A major and long-standing problem affecting stellar model computations is the treatment of superadiabatic convection. This lack prevents a firm and reliable prediction of the extension of the external convective regions.

A precise treatment of external convection would require 3D hydrodynamical calculations, greatly improved in recent years (see e.g., Tanner et al., 2013; Trampedach et al., 2014; Magic et al., 2015); however, they still cannot cover the wide range of input physics needed to model stellar computations. Moreover, defining alpha in one-dimensional codes from the results of $3 \mathrm{D}$ simulations is quite ambiguous due to the differences between the mixing length representation of convection and the convection behavior in 2D/3D simulations, even if there have been relevant attempts in the literature (Lydon et al., 1992; Ludwig et al., 1999; Tanner et al., 2013; Magic et al., 2015; Mosumgaard et al., 2017). Therefore, the current generation of stellar evolution codes still addresses this problem by relying, almost universally, on the mixing-length theory (Böhm-Vitense, 1958). In this framework, the efficiency of the convective transport and the stellar structure in the superadiabatic transition layers depends on the mixinglength $l$, which is supposed to be proportional to the pressure scale height $H_{p}$, i.e., $l=\alpha_{\mathrm{ml}} H_{p}$, where $\alpha_{\mathrm{ml}}$ is a non-dimensional free parameter to be somehow calibrated.

As a result of this freedom, neither the effective temperature nor the radius of stars with a thick outer convective envelope can be firmly predicted by the current generation of $1 \mathrm{D}$ stellar models since they strongly depend on the calibrated value of $\alpha_{\mathrm{ml}}$. This obviously influences the stellar characteristics recovered by fit techniques that exploit these observables.

The classical target for mixing-length calibration is the Sun, but the generalization of this calibration to different evolutionary phases, metallicity, and mass ranges has been questioned both on theoretical and observational grounds. In particular, a growing amount of observations suggests that the adoption of the solar calibrated $\alpha_{\mathrm{ml}}$ does not allow proper modeling of all kinds of stars (see e.g., Guenther and Demarque, 2000; Yildiz et al., 2006; Yild1z, 2007; Clausen et al., 2009; Deheuvels and Michel, 2011; Bonaca et al., 2012; Mathur et al., 2012; Wu et al., 2015; Joyce and Chaboyer, 2018a,b; Li et al., 2018).

Several of the above mentioned studies consider stars in double-lined detached eclipsing binary systems. In fact, in this case it is possible to obtain an accurate measurement of both masses and radii of the two stars. The availability of these two fundamental quantities allows for stringent tests of the stellar models (see e.g., Claret, 2007; Stancliffe et al., 2015; Claret and Torres, 2016, 2017; Gallenne et al., 2016; Valle et al., 2017) and thus also on the possible variations of the mixing-length parameter in different mass and metallicity ranges.

A recent theoretical analysis (Valle et al., 2019) pointed out many limitations for a mixing-length calibration from field stars, so it is interesting to investigate the question of the reliability of this calibration in binary systems. The aim of this work is to perform such an investigation for a system composed of two low mass stars, thus avoiding the supplementary complication of dealing with the concurrent calibration of the convective core overshooting parameter.

The investigation is focused on a synthetic binary system composed by a primary of $M_{1}=0.95 M_{\odot}$ and a secondary of $M_{2}=0.85 M_{\odot}$, resulting in a mass ratio of about 1.1 . This configuration, quite common for real systems, was chosen also to allow the stars to be sampled in different evolutionary phases, because this provides the most stringent constraints for the recovery (Claret and Torres, 2016; Valle et al., 2017). The focus of our analysis is in quantifying the errors in the recovered mixinglength values arising only from observational errors. Therefore, no systematic discrepancies between the grid of models adopted in the recovery and the artificial stars are assumed. Although this is a far too optimistic assumption when dealing with real-world binary systems, it is nevertheless a mandatory step that allows one to assess the very minimum amount of errors that can be expected in the calibration process. Moreover, only a theoretical investigation can highlight the presence of hidden biases and dependencies (see e.g., Valle et al., 2019, for an analysis of spurious metallicity dependencies of the recovered mixing-length on field stars) that would otherwise be neglected.

\section{METHODS}

The analysis was performed at three different metallicity values, centered around the solar value $(Z=0.0074,0.0129,0.0221)$ and at three different evolutionary stages of the primary star. More precisely, we define a relative age $r$ with respect to the main sequence (MS) lifetime, and we selected models at $r=60,90$, and $120 \%$ of the central hydrogen exhaustion time. The first two points correspond to a primary at the middle and nearly at the end of the MS, while the third one corresponds to a primary in the red giant branch (RGB) phase. Correspondingly, the secondary star is always in the MS, at about 40,55 , and $75 \%$ of its central hydrogen exhaustion time. The synthetic stars' initial helium abundances were obtained by the linear relation $Y=Y_{p}+\frac{\Delta Y}{\Delta Z} Z$, 
with the primordial abundance $Y_{p}=0.2485$ from WMAP (Peimbert et al., 2007a,b) and with a helium-to-metal enrichment ratio of $\Delta Y / \Delta Z=2.0$ (see e.g., Gennaro et al., 2010).

We considered different possible values of $\alpha_{\mathrm{ml}}$ for the two stars. The reference scenario has the two stars with a common mixing-length parameter $\alpha_{\mathrm{ml}, 1}=\alpha_{\mathrm{ml}, 2}=2.0$. The other five cases adopted a systematically increasing difference between the two mixing-length values at a step of 0.2 ; while the mixing length of the primary star was increased at a step of $0.1\left(\alpha_{\mathrm{ml}, 1}=2.1\right.$, $2.2,2.3,2.4$, and 2.5$)$, the values of $\alpha_{\mathrm{ml}, 2}$ were correspondingly decreased by $0.1\left(\alpha_{\mathrm{ml}, 2}=1.9,1.8,1.7,1.6,1.5\right)$. Thus, the extreme scenario has a difference in mixing-length value $\alpha_{\mathrm{ml}, 1}-\alpha_{\mathrm{ml}, 2}$ $=1.0$. The considered simulation parameters are summarized in Table 1.

For each possible mixing-length combination, metallicity and evolutionary stage artificial stars were sampled from the grid described in section 2.2, and their observables were perturbed by means of a Monte Carlo procedure assuming Gaussian errors. The procedure was repeated $N=5,000$ times for each artificial system. The adopted observable constraints were the effective temperature $T_{\text {eff, metallicity }}[\mathrm{Fe} / \mathrm{H}]$, mass $M$, and radius $R$ of both stars. We assumed uncertainties of $100 \mathrm{~K}$ in $T_{\text {eff }}, 0.1$ $\operatorname{dex}$ in $[\mathrm{Fe} / \mathrm{H}], 0.5 \%$ in $M$ and $0.25 \%$ in $R$. Similar precisions on the mass and radii are achievable for a small subset of binary systems, but they are mandatory for calibration purposes (see e.g., Valle et al., 2017).

\subsection{Grid-Based Recovery Technique}

Each artificial system was then reconstructed adopting the SCEPtER-binary pipeline (Valle et al., 2015a), modified to consider the mixing-length value in the estimation process. Details about the technique can be found in Valle et al. (2015a, 2016).

We adopted the pipeline in three configurations. The first one fits the two stars independently, thus effectively losing the binary constraint. This result is useful as a reference for comparison with the other results. A second configuration imposes the binary constraint, forcing the pipeline to return identical ages, initial metallicity $Z$, and initial helium abundance $Y$ for the two stars; however, each star can have different mixing-length values. The third configuration adds the constraint of a common mixinglength value. The latter scenario is particularly useful to test the sensitivity of grid techniques in identifying an inadequate fitting model specification. Indeed, as the difference in the mixing length of the synthetic stars grows, the constraint of a common $\alpha_{\mathrm{ml}}$ value

TABLE 1 | Summary of the simulation parameters for the considered synthetic binary systems.

\begin{tabular}{lcc}
\hline Parameter & Primary & Secondary \\
\hline Mass $\left(M_{\odot}\right)$ & 0.95 & 0.85 \\
Relative ages & $0.60,0.90,1.20$ & $0.38,0.57,0.76$ \\
$\alpha_{\mathrm{ml}}$ & $2.0,2.1,2.2,2.3,2.4,2.5$ & $2.0,1.9,1.8,1.7,1.6,1.5$ \\
$Z$ & $0.0074,0.0129,0.0221$ & $0.0074,0.0129,0.0221$ \\
$\Delta Y / \Delta Z$ & 2.0 & 2.0
\end{tabular}

would become more and more difficult to satisfy, causing several fits to return no acceptable values for the system. The adopted procedure will allow quantification of the theoretical fraction of systems for which such behavior is expected.

\subsection{Stellar Models Grid}

The estimation procedure required a grid of stellar models, sufficiently extended to cover the whole parameter space. To this purpose, we adopted the same stellar model grid as in Valle et al. (2019), computed by means of the FRANEC code (Degl'Innocenti et al., 2008; Tognelli et al., 2011), in the same configuration as was adopted to compute the Pisa Stellar Evolution Data Base ${ }^{1}$ for low-mass stars (Dell'Omodarme et al., 2012). Models were calculated for the solar heavy-element mixture by Asplund et al. (2009). Atomic diffusion was included, taking into account the effects of gravitational settling and thermal diffusion with coefficients given by Thoul et al. (1994). Outer boundary conditions were determined by integrating the $T(\tau)$ relation by Krishna Swamy (1966). Further details on the stellar models can be found in Valle et al. (2009, 2015a,b) and the references therein. Although the choices in the input physics play a relevant role when estimating stellar parameters from real observational data, they are of minor relevance for our aim because artificial stars are recovered from the same model grid adopted for their sampling.

The adoption of microscopic diffusion in the stellar computations causes an evolution with time of the surface $[\mathrm{Fe} / \mathrm{H}]$, which is adopted as one of the observational constraints in the analysis. Figure 1 shows the trend of the surface $[\mathrm{Fe} / \mathrm{H}]$ as a function of the relative age. As is well known, the $[\mathrm{Fe} / \mathrm{H}]$ abundance reaches a minimum around the end of the MS, while it increases at later time due to the first dredge-up.

The grid of models spans the range $[0.8,1.0] M_{\odot}$, with a step of $0.01 M_{\odot}$, and covers the initial metallicity interval -0.4 dex $\leq$ $[\mathrm{Fe} / \mathrm{H}] \leq 0.4 \mathrm{dex}$, with a step of 0.05 dex. For each metallicity, we computed models for nine different values of the initial helium abundance by following the above mentioned linear relation $Y=Y_{p}+\frac{\Delta Y}{\Delta Z} Z$, with a helium-to-metal enrichment ratio $\Delta Y / \Delta Z$ from 1 to 3 with a step of 0.25 (Gennaro et al., 2010). $\Delta Y / \Delta Z=2.0$ corresponds to the reference value for the synthetic systems. Ultimately, the grid spans a set of 153 different initial chemical compositions. For each mass, metallicity and initial helium abundance, we computed models for 21 values of the mixing-length parameter $\alpha_{\mathrm{ml}}$ in the range $[1.0,3.0]$ with a step of 0.1 . With the assumed input physics, the solar-calibrated value is $\alpha_{\mathrm{ml}}=2.1$. All the adopted steps are sufficiently small to have a negligible impact on the estimates.

\section{RESULTS}

The analysis of the fit results for the considered scenarios revealed some expected behaviors and also some peculiar effects. The following subsections explore in detail the outcomes in the three considered fitting configurations.

\footnotetext{
${ }^{1}$ http://astro.df.unipi.it/stellar-models/
} 


\subsection{Independent Recovery}

The fit of the binary systems under full independence between the stars (i.e., stars can be fitted at different chemical compositions and different ages) revealed a great difficulty to constrain the mixing-length value for the two stars. The recovered marginalized posterior densities of $\alpha_{\mathrm{ml}, 1}$ and $\alpha_{\mathrm{ml}, 2}$ are presented in Figure 2 and Table 2. While the mean values of the mixinglength is in general consistently recovered for both stars, there is a huge variability in the results, which practically cover the whole allowed $\alpha_{\mathrm{ml}}$ range. The $\alpha_{\mathrm{ml}, 1}$ values are underestimated for the two most extreme scenarios $\left(\alpha_{\mathrm{ml}, 1}=2.4,2.5\right)$ as a consequence

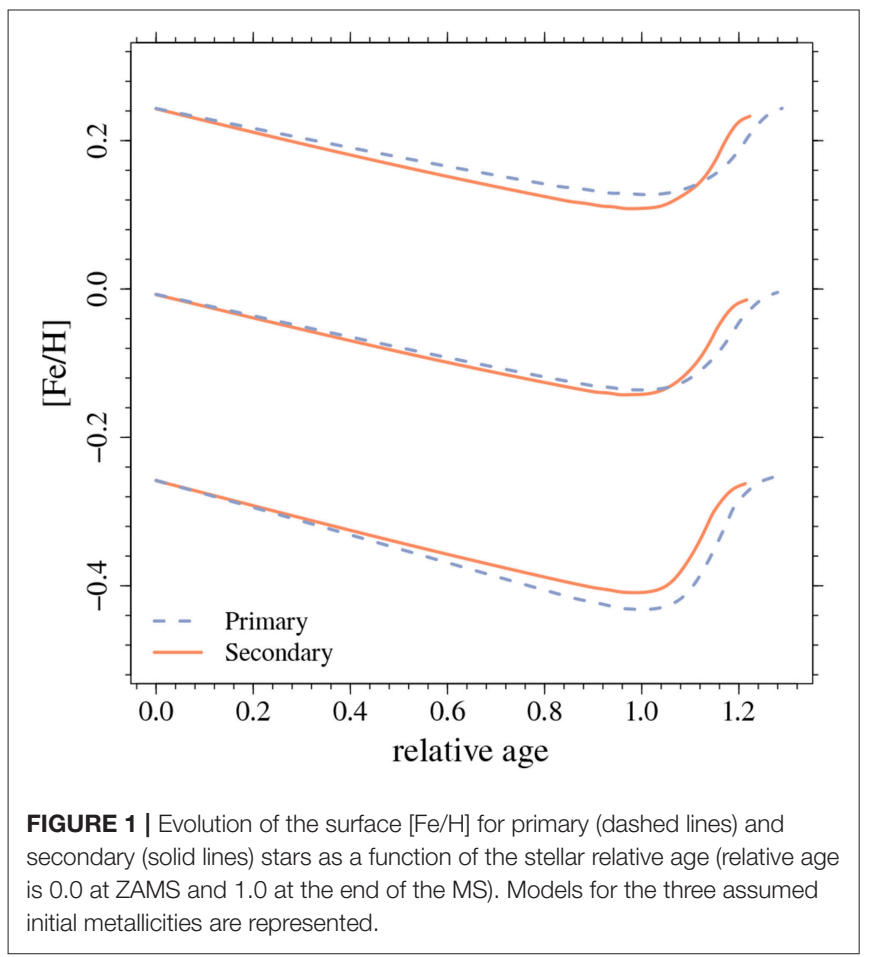

TABLE 2 | Mean and standard deviation of the recovered mixing-length values for primary and secondary stars, depending on the $\Delta \alpha_{\mathrm{ml}}$ adopted in the generation.

\begin{tabular}{|c|c|c|c|c|c|c|}
\hline \multirow[b]{2}{*}{$\Delta \alpha_{\mathrm{ml}}$} & \multirow[b]{2}{*}{$\alpha_{\mathrm{ml}, 1}$} & \multirow[b]{2}{*}{$\alpha_{\mathrm{ml}, 2}$} & \multicolumn{2}{|c|}{ Primary } & \multicolumn{2}{|c|}{ Secondary } \\
\hline & & & Mean & $S D$ & Mean & $S D$ \\
\hline 0.0 & 2.0 & 2.0 & 2.03 & 0.41 & 2.06 & 0.40 \\
\hline 0.2 & 2.1 & 1.9 & 2.12 & 0.41 & 1.97 & 0.39 \\
\hline 0.4 & 2.2 & 1.8 & 2.20 & 0.41 & 1.87 & 0.38 \\
\hline 0.6 & 2.3 & 1.7 & 2.28 & 0.41 & 1.77 & 0.36 \\
\hline 0.8 & 2.4 & 1.6 & 2.36 & 0.40 & 1.67 & 0.35 \\
\hline 1.0 & 2.5 & 1.5 & 2.44 & 0.39 & 1.56 & 0.32 \\
\hline
\end{tabular}

The reference $\alpha_{m l}$ of the synthetic data are listed in the second and third columns.

of an edge effect that truncates the estimates at the grid upper value $\left(\alpha_{\mathrm{ml}}=3.0\right)$. This effect is clearly evidenced in Figure 2A; for the sampling at $\alpha_{\mathrm{ml}}=2.5$ the posterior density is clearly truncated at the upper edge. A corresponding tendency toward overestimation is reported in the last two cases for the secondary star $\left(\alpha_{\mathrm{ml}, 2}=1.6\right.$ and 1.5). These results confirm the theoretical finding by Valle et al. (2019), obtained for field stars. It seems that even from stars with exceptionally well constrained masses and radii, a calibration of the mixing-length parameter is not very reliable.

\subsection{Binary Constraints in Age and Chemical Composition}

Imposing the constraint of a common age and initial chemical composition for the two stars modifies the results in an interesting way. As it appears from Figure 3 and Table 3, the recovered $\alpha_{\mathrm{ml}, 1}$ values closely match the ones shown in section 3.1, showing the same mean values and the characteristic large dispersion. On the other hand, the recovered $\alpha_{\mathrm{ml}, 2}$ values are much more peaked around their mean values, which provide unbiased estimates of the values adopted in the sampling. These
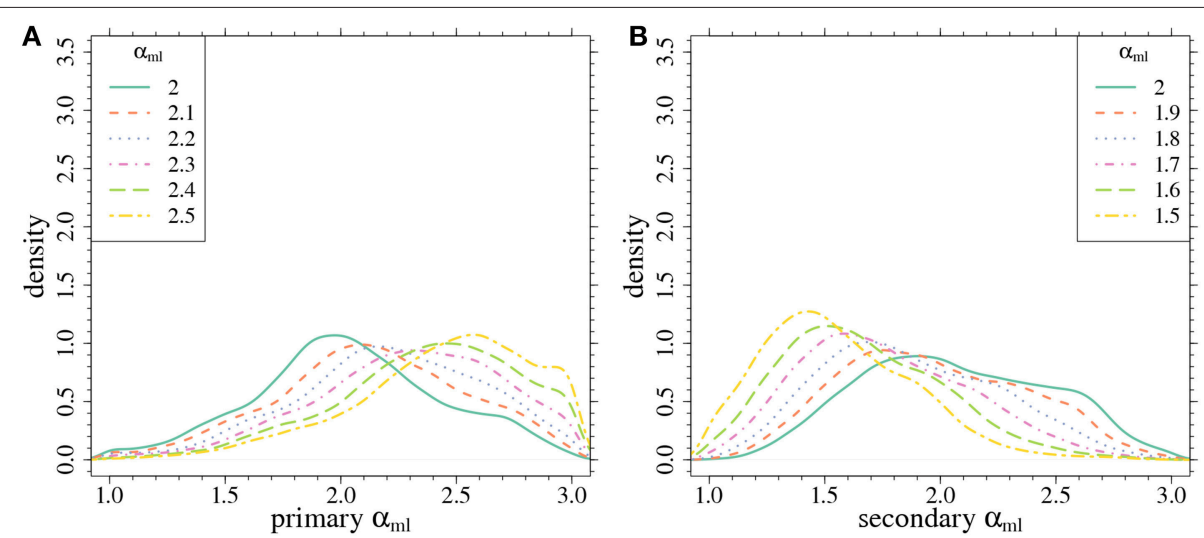

FIGURE 2 | (A): density of probability of mixing-length value estimates for the primary star, for the independent stellar fit. Different line styles identify the $\alpha_{\mathrm{ml}}$ values from (2.0 to 2.5) adopted in the sampling of the mock data. (B): same as in the (A) panel, but for the secondary star. The sampling $\alpha_{\mathrm{ml}}$ values for the secondary star run from 2.0 to 1.5 . 

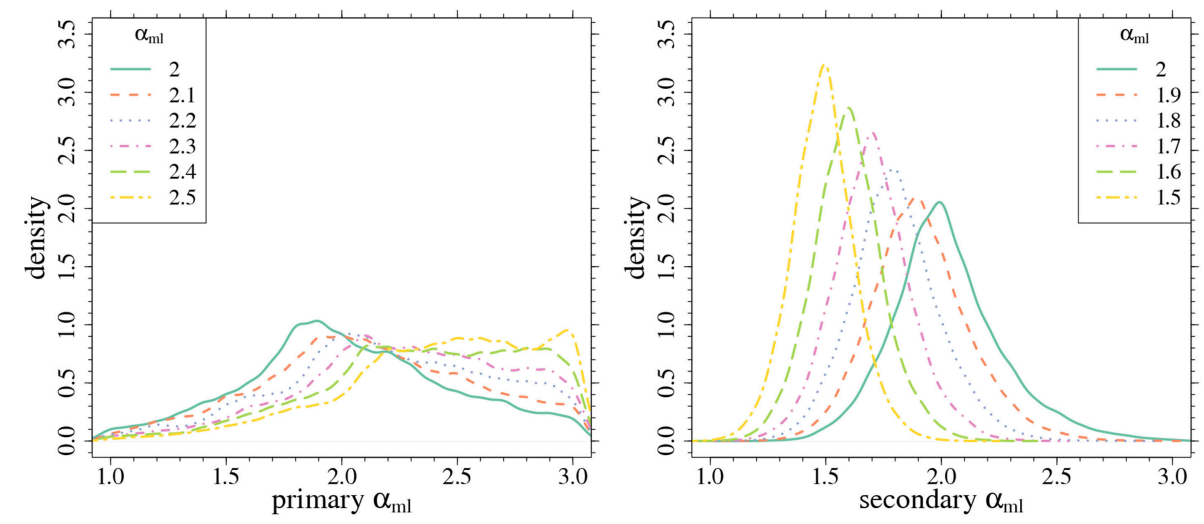

FIGURE 3 | As in Figure 2, but assuming common age, and common initial $Z$ and $Y$ in the fit.

TABLE 3 | As in Table 2, but imposing the constraints of common age, and common initial $Z$ and $Y$ for the binary components.

\begin{tabular}{llllll}
\hline & \multicolumn{2}{c}{ Primary } & & \multicolumn{2}{c}{ Secondary } \\
\cline { 2 - 3 } $\boldsymbol{n n n n n} \boldsymbol{n n n n n}$ & Mean & SD & & Mean & SD \\
\hline 0.0 & 2.03 & 0.44 & & 2.02 & 0.24 \\
0.2 & 2.11 & 0.46 & & 1.91 & 0.21 \\
0.4 & 2.19 & 0.46 & & 1.80 & 0.19 \\
0.6 & 2.28 & 0.44 & & 1.70 & 0.16 \\
0.8 & 2.35 & 0.44 & & 1.60 & 0.15 \\
1.0 & 2.43 & 0.42 & & 1.49 & 0.13 \\
\hline
\end{tabular}

results suggest that while the mixing-length parameter of the primary star is not further constrained by the fit, this is not the case for that of the secondary. Indeed, the standard deviations of the recovered $\alpha_{\mathrm{ml}, 2}$ values range from one half to one third of those of $\alpha_{\mathrm{ml}, 1}$. Moreover, the tendency to overestimate $\alpha_{\mathrm{ml}, 2}$ for the two most extreme scenarios-shown in section 3.1disappears as a consequence of the much smaller variability which prevents edge effects from playing a role.

The impressive differences in the two stars' behavior are dictated by the different constraints they provide each other in the joint fit. While the independently recovered initial chemical compositions are nearly identical for both stars, this is not the case for the age. As discussed in detail in Valle et al. (2015a), the primary star provides a much tighter age constraint in the joint fit than in the secondary. This occurs because the agerelative error becomes smaller as a star evolves in the MS, due to the faster evolutionary time scale. In fact, the age range allowed by the observable constraint errors is smaller in rapid evolutionary phases. This is clearly demonstrated in Figure 4, which presents the $\alpha_{\mathrm{ml}, 2}$ posterior density for mock data as $\alpha_{\mathrm{ml}, 1}=\alpha_{\mathrm{ml}, 2}=2.0$, depending on the evolutionary stage of the primary star. The distribution of the recovered secondary mixing-length values shrinks as the primary evolves. The RGB scenario-for which the evolutionary time scale is the fastestprovides the lowest variance for the estimated $\alpha_{\mathrm{ml}, 2}$ values.
This effect is further shown in Table 4. It is apparent that the standard deviation of the recovered mixing-length value for the secondary star shrinks as the primary evolves, mainly when the differences in the sampled $\alpha_{\mathrm{ml}}$ value are lower than about 0.6.

As a consequence, the common age constraint leads to the rejection of several extreme solutions for the secondary, while the solutions of the primary are unaffected. The net result is a selection on the mixing-length values of the secondary star, which disfavors extreme variations with respect to the sampling values.

\subsubsection{Expected Difference in $\alpha_{\mathrm{ml}}$ Value for the Two Stars}

The analysis conducted assuming independent mixing-length values for the two stars also allows for the estimation of an interesting parameter, which is the expected dispersion of the recovered $\alpha_{\mathrm{ml}}$ values when sampled at common $\alpha_{\mathrm{ml}}=2.0$. An estimate of this value can help in judging how reliable the calibrations from binary systems are, allowing for independent mixing-length values.

The question requires considering the reconstructed differences $\Delta \alpha_{\mathrm{ml}}=\alpha_{\mathrm{ml}, 1}-\alpha_{\mathrm{ml}, 2}$ for all the binary systems simulated with the same $\alpha_{\mathrm{ml}}$. The distribution of these differences is only due to random errors on the observables and should therefore be considered as the minimum variability on the mixing-length parameter. Figure 5 shows the estimated distribution of $\Delta \alpha_{\mathrm{ml}}$ with the identification of the expected $1 \sigma$ and $2 \sigma$ quantiles. It appears that a fluctuation of $\Delta \alpha_{\mathrm{ml}} \pm 0.3$ is expected at $1 \sigma$ levels, implying that about $32 \%$ of the systems with true common $\alpha_{\text {ml }}$ values can be reconstructed with differences higher than this only owing to the observational errors.

These results should be carefully considered as a difference of 0.3 in $\alpha_{\mathrm{ml}}$ is as high as $15 \%$ of the solar-scaled value. For investigations that report a difference in mixing-length values of two stars in a binary system lower than this, one should consider the possibility that a random error on the observables can indeed explain this discrepancy. 


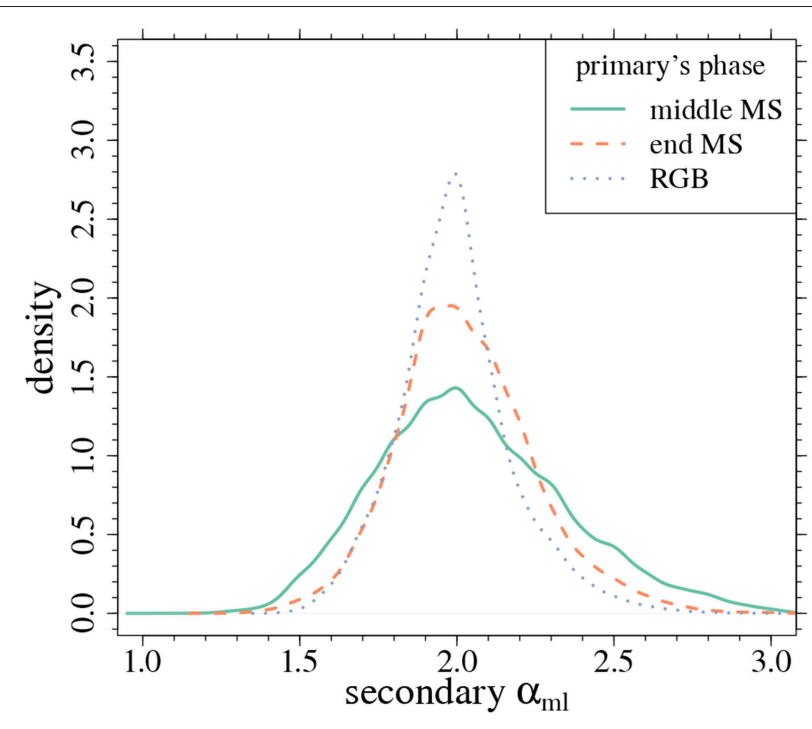

FIGURE 4 | Density of probability of mixing-length value estimates for the secondary star for synthetic data with $\alpha_{\mathrm{ml}}=2.0$, as a function of the evolutionary phase of the primary star.

TABLE 4 | As in Table 3, but splitting the result according to the evolutionary phase of the primary star.

\begin{tabular}{|c|c|c|c|c|c|c|c|}
\hline \multirow[t]{3}{*}{$\Delta \alpha_{\mathrm{ml}}$} & \multirow[t]{3}{*}{$\alpha_{\mathrm{ml}}$} & Mean & $S D$ & Mean & $S D$ & Mean & $S D$ \\
\hline & & \multicolumn{6}{|c|}{ Primary } \\
\hline & & \multicolumn{2}{|c|}{$r=0.6$} & \multicolumn{2}{|c|}{$r=0.9$} & \multicolumn{2}{|c|}{$r=1.2$} \\
\hline 0.0 & 2.0 & 2.08 & 0.42 & 2.08 & 0.36 & 1.93 & 0.52 \\
\hline 0.2 & 2.1 & 2.16 & 0.41 & 2.17 & 0.36 & 2.00 & 0.55 \\
\hline 0.4 & 2.2 & 2.24 & 0.41 & 2.25 & 0.36 & 2.08 & 0.56 \\
\hline 0.6 & 2.3 & 2.31 & 0.39 & 2.35 & 0.36 & 2.17 & 0.54 \\
\hline 0.8 & 2.4 & 2.39 & 0.38 & 2.42 & 0.35 & 2.23 & 0.54 \\
\hline \multirow[t]{2}{*}{1.0} & 2.5 & 2.45 & 0.37 & 2.49 & 0.34 & 2.34 & 0.52 \\
\hline & & \multicolumn{6}{|c|}{ Secondary } \\
\hline 0.0 & 2.0 & 2.05 & 0.30 & 2.03 & 0.22 & 2.00 & 0.18 \\
\hline 0.2 & 1.9 & 1.92 & 0.26 & 1.91 & 0.19 & 1.89 & 0.17 \\
\hline 0.4 & 1.8 & 1.82 & 0.22 & 1.80 & 0.17 & 1.79 & 0.16 \\
\hline 0.6 & 1.7 & 1.69 & 0.19 & 1.71 & 0.16 & 1.71 & 0.14 \\
\hline 0.8 & 1.6 & 1.59 & 0.17 & 1.60 & 0.14 & 1.60 & 0.13 \\
\hline 1.0 & 1.5 & 1.47 & 0.14 & 1.49 & 0.12 & 1.50 & 0.12 \\
\hline
\end{tabular}

The relative age $r$ assumes value 0.0 at ZAMS and 1.0 at the end of the MS.

\subsection{Fully Coupled Recovery}

The last explored reconstruction also imposes the constraint of a common mixing-length value for the two stars, beside those on the common original chemical composition and age, as discussed in section 3.2. In nature, a similar assumption is theoretically justified when stars have a similar mass and are in the same evolutionary phase, but can otherwise be questioned. For our mock data, this assumption is not valid for most of the systems, which are sampled with different mixing-length values for the two stars. The assumption of a common $\alpha_{\mathrm{ml}}$ is thus useful as

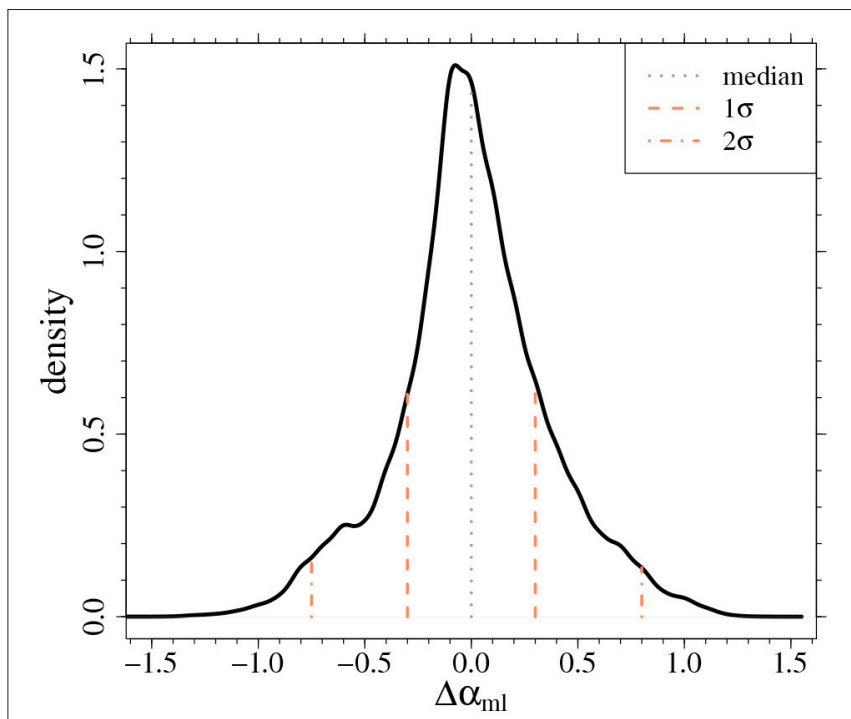

FIGURE 5 | Distribution of the expected differences between the recovered mixing-length values of the primary and secondary star, when both stars in the synthetic systems have common $\alpha_{\mathrm{ml}}=2.0$.

a test of the robustness of the fit to wrong assumptions in the mixing-length values.

As shown in Figure 6 and in Table 5, the reconstructed mixing-length values cluster around the value of the secondary star, with a low variability. This phenomenon is easily comprehensible in light of the discussion in section 3.2. The posterior density of the secondary is much more peaked than that of the primary, thus providing a much stronger constraint on the joint estimate.

In the extreme scenario of $\Delta \alpha_{\mathrm{ml}}=1.0$ the mixing-length value of the primary is underestimated from 2.5 to 1.5. This severe underestimation comes at one cost; only a marginal share of systems in this configuration can be reconstructed by the algorithm. For the vast majority, the fitting pipeline is not able to provide an acceptable fit, thus suggesting the existence of some wrong assumptions in the modeling. Indeed, Figure 6B shows the fraction of systems for which a fit was possible. While a common mixing-length value is found for more than three quarters of systems with $\Delta \alpha_{\mathrm{ml}} \leq 0.4$, this fraction rapidly drops to $20 \%$ for $\Delta \alpha_{\mathrm{ml}}=1.0$. Moreover, restricting the analysis to systems with both stars in the MS, the fraction of systems for which the fit was possible decreases even more and is nearly zero for $\Delta \alpha_{\mathrm{ml}}=1.0$.

Therefore, it seems that large discrepancies between the $\alpha_{\mathrm{ml}}$ assumptions in the fit and in the mock data are easily detected. This is not the case for moderate differences: in this case, the fitting algorithm is able to provide a common solution, which is however biased toward $\alpha_{\mathrm{ml}, 2}$.

\subsection{The Impact of the Observational Error in the Effective Temperature}

The results presented in the previous sections assume a fixed error on the observational constraints. It is interesting to explore how these assumptions influence the outcome of the fit in the 

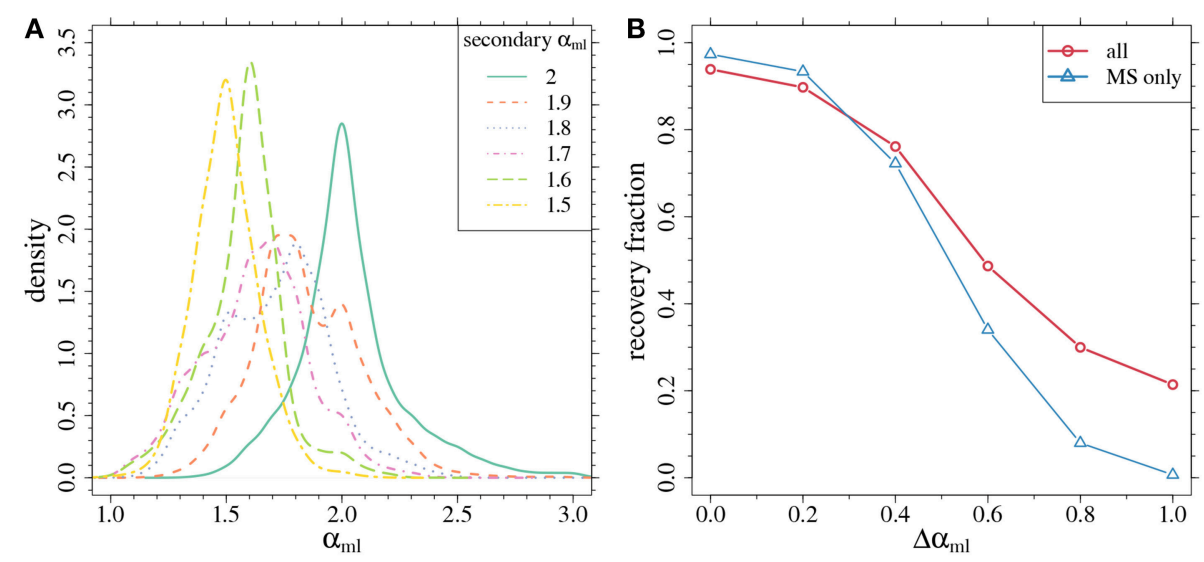

FIGURE 6 | (A): density of probability of the recovered mixing-length value when a common $\alpha_{\mathrm{ml}}$ is imposed in addition to the constraint of common age and original chemical composition. (B): fraction of systems with a valid mixing-length value fit. The circles identify all the solutions, while triangles correspond only to MS cases.

TABLE 5 | Means and standard deviations of the recovered mixing-length values in the full coupled scenario, depending on the $\Delta \alpha_{\mathrm{ml}}$ adopted in the generation.

\begin{tabular}{llccc}
\hline $\boldsymbol{\Delta} \boldsymbol{\alpha}_{\mathrm{ml}}$ & $\boldsymbol{\alpha}_{\mathrm{ml}, \mathbf{1}}$ & $\boldsymbol{\alpha}_{\mathrm{ml}, \mathbf{2}}$ & Mean & SD \\
\hline 0.0 & 2.0 & 2.0 & 2.03 & 0.23 \\
0.2 & 2.1 & 1.9 & 1.85 & 0.23 \\
0.4 & 2.2 & 1.8 & 1.72 & 0.23 \\
0.6 & 2.3 & 1.7 & 1.63 & 0.23 \\
0.8 & 2.4 & 1.6 & 1.58 & 0.17 \\
1.0 & 2.5 & 1.5 & 1.50 & 0.14 \\
\hline
\end{tabular}

three explored configurations. Due to the strong dependence of the effective temperature on the adopted mixing-length, we repeated the analysis assuming an error of $50 \mathrm{~K}$ in $T_{\text {eff }}$, which is one half of what was previously assumed.

The results are summarized in Table 6. A comparison with Tables 2, 3, 5 shows a minor impact of this change. Overall, one can observe a moderate reduction of the standard deviations, and an equally small reduction of the biases in the recovered mixing-length values. Overall, the reduction of the standard deviation of the recovered mixing-length values in the three scenarios is about $20 \%$, with respect to a $50 \%$ reduction of the $T_{\text {eff }}$ uncertainty. Therefore, the results and trends discussed so far can be considered robust against this particular source of uncertainty.

\section{CONCLUSIONS}

We performed a theoretical investigation on the biases and random uncertainties affecting the calibration of the mixinglength value from a mock eclipsing double-lined binary system, composed of a primary artificial star of mass $M_{1}=0.95 M_{\odot}$ and a secondary of mass $M_{2}=0.85 M_{\odot}$. We used the SCEPtER-binary pipeline (Valle et al., 2015a) to estimate the mixing-length of the mock stars, adopting as observational constraint the effective temperature, the metallicity $[\mathrm{Fe} / \mathrm{H}]$, the radius, and the mass of the two stars. The comparison between the true and the estimated mixing-length values allows to evaluate the calibration reliability.

More in detail, several Monte Carlo simulations were conducted considering nine different scenarios, consisting of three metallicities, coupled to three different evolutionary stages of the primary $(0.6,0.9$ and 1.2 of the central hydrogen exhaustion time). For each configuration, data were sampled assuming an increasing difference between the mixing-length of the two stars, from perfect agreement at $\alpha_{\mathrm{ml}, 1}=\alpha_{\mathrm{ml}, 2}=2.0$ to a maximum difference of $1.0\left(\alpha_{\mathrm{ml}, 1}=2.5, \alpha_{\mathrm{ml}, 2}=1.5\right)$.

The mixing-length values were then estimated adopting a different hypothesis in the recovery procedure. In the first case we assumed full independence between the two stars and reconstructed them without imposing any constraint in age and chemical composition between the stars. Great difficulty to estimate the mixing-length value for the two stars resulted under these hypotheses. The standard deviation of the recovered values was about 0.40 , confirming the difficulties pointed out for field stars in Valle et al. (2019). Thus, even from stars with exceptionally well constrained masses and radii, the calibration of the mixing-length parameter seems unreliable.

In the second case, we imposed the constraint of common age and initial chemical composition for the two stars in the recovery. While the fitted $\alpha_{\mathrm{ml}, 1}$ values closely match those recovered under full independence, the $\alpha_{\mathrm{ml}, 2}$ values are much more peaked around unbiased estimates of the values adopted in the sampling. The standard deviations of the recovered $\alpha_{\mathrm{ml}, 2}$ values range from one half to one third of those of $\alpha_{\mathrm{ml}, 1}$. This occurs because the primary star provides a much tighter age constraint in the joint fit than the secondary. This leads to the rejection of several extreme solutions for the secondary, while the solutions of the primary are unaffected. In this scenario, we also explored the difference in the mixing-length values of the two stars due to random fluctuations owing to observational errors. We considered stars sampled at common $\alpha_{\mathrm{ml}}=2.0$ and focused the analysis on the distribution of the differences $\alpha_{\mathrm{ml}, 1}-\alpha_{\mathrm{ml}, 2}$. We found that the posterior distribution of these differences peaked around zero, with a somewhat large standard deviation of 0.3 (about 
TABLE 6 | Means and standard deviations of the recovered mixing-length values adopting an observational error of $50 \mathrm{~K}$ in $T_{\text {eff }}$.

\begin{tabular}{|c|c|c|c|c|c|c|c|c|c|c|c|}
\hline \multirow[b]{3}{*}{$\alpha_{\mathrm{ml}, 1}$} & \multirow[b]{3}{*}{$\alpha_{\mathrm{ml}, 2}$} & \multicolumn{4}{|c|}{ Independent } & \multicolumn{4}{|c|}{ Coupled } & \multicolumn{2}{|c|}{ Fully coupled } \\
\hline & & \multicolumn{2}{|c|}{ Primary } & \multicolumn{2}{|c|}{ Secondary } & \multicolumn{2}{|c|}{ Primary } & \multicolumn{2}{|c|}{ Secondary } & \multirow[b]{2}{*}{ Mean } & \multirow[b]{2}{*}{$S D$} \\
\hline & & Mean & $S D$ & Mean & $S D$ & Mean & $S D$ & Mean & $S D$ & & \\
\hline 2.0 & 2.0 & 2.02 & 0.34 & 2.06 & 0.34 & 2.00 & 0.36 & 2.03 & 0.22 & 2.03 & 0.22 \\
\hline 2.1 & 1.9 & 2.12 & 0.35 & 1.97 & 0.33 & 2.09 & 0.38 & 1.91 & 0.19 & 1.91 & 0.19 \\
\hline 2.2 & 1.8 & 2.20 & 0.34 & 1.86 & 0.32 & 2.18 & 0.38 & 1.81 & 0.17 & 1.81 & 0.17 \\
\hline 2.3 & 1.7 & 2.29 & 0.34 & 1.76 & 0.30 & 2.29 & 0.37 & 1.71 & 0.15 & 1.71 & 0.15 \\
\hline 2.4 & 1.6 & 2.38 & 0.33 & 1.65 & 0.28 & 2.37 & 0.36 & 1.60 & 0.13 & 1.60 & 0.13 \\
\hline 2.5 & 1.5 & 2.45 & 0.32 & 1.54 & 0.26 & 2.46 & 0.35 & 1.49 & 0.11 & 1.49 & 0.11 \\
\hline
\end{tabular}

$15 \%$ of the solar-scaled value). Therefore, about $32 \%$ of systems with true identical $\alpha_{\mathrm{ml}}$ are expected to show differences higher than that caused only by random errors. These results should be carefully considered when obtaining a fit from a real binary system, because a difference lower than this has a great chance to be only a random fluctuation.

In the third case we also imposed the constraint of common mixing-length value for the two stars, beside those on chemical composition and age. Two interesting effects were detected. First, the estimated common mixing-length is mainly dictated by the value of $\alpha_{\mathrm{ml}, 2}$. This happens because, as discussed above, the posterior distribution of the mixing-length of the secondary star under partial independence is much more peaked than that of the primary, thus dominating in the joint estimate process. Second, an increasing share of systems cannot be fitted by the algorithm as the differences between the true $\alpha_{\mathrm{ml}}$ increase. For $\Delta \alpha_{\mathrm{ml}}>0.4$ less than half of the systems can be recovered; at $\Delta \alpha_{\mathrm{ml}}=1.0$ the values decreases at $20 \%$. Therefore, it seems that moderate differences in the mixing-length value between the two stars are difficult to detect and in these cases the solution is biased toward $\alpha_{\mathrm{ml}, 2}$.

While most of the results presented in this paper can be considered general, such as the effect of the shrink of the estimated mixing-length value around the value of the secondary

\section{REFERENCES}

Asplund, M., Grevesse, N., Sauval, A. J., and Scott, P. (2009). The chemical composition of the sun. Ann. Rev. Astron. Astrophys. 47, 481-522. doi: 10.1146/annurev.astro.46.060407.145222

Böhm-Vitense, E. (1958). Über die Wasserstoffkonvektionszone in Sternen verschiedener Effektivtemperaturen und Leuchtkräfte. Mit 5 Textabbildungen. zap 46:108.

Bonaca, A., Tanner, J. D., Basu, S., Chaplin, W. J., Metcalfe, T. S., Monteiro, M. J. P. F. G., et al. (2012). Calibrating convective properties of solar-like stars in the kepler field of view. Astrophys. J. Lett. 755:L12. doi: $10.1088 / 2041-8205 / 755 / 1 / \mathrm{L} 12$

Claret, A. (2007). Does convective core overshooting depend on stellar mass? Tests using double-lined eclipsing binaries. Astron. Astrophys. 475, 1019-1025. doi: 10.1051/0004-6361:20078024

Claret, A., and Torres, G. (2016). The dependence of convective core overshooting on stellar mass. Astron. Astrophys. 592:A15. doi: 10.1051/0004-6361/201628779 star, nonetheless this work deals only with a specific binary system with fixed masses. Therefore, the quantitative results cannot be expected to be valid without modifications for other binary systems with different masses and in different evolutionary phases. Although checking the robustness of the results presented here for different ranges of mass or by adopting a different mass ratio would be highly desirable, this possibility is actually limited by the very large computational burden required to compute the stellar models for the recovery at the required level of accuracy. For these reasons, our study should be considered as a first step in this exploration, adopting a quite common value of masses, and mass ratio. More theoretical investigations are required to fully address this open topic.

\section{AUTHOR CONTRIBUTIONS}

GV and MD computed the stellar models grid and performed the recovery. GV, MD, PP, and SD wrote the manuscript.

\section{FUNDING}

This work has been supported by PRA Università di Pisa 20182019 (Le stelle come laboratori cosmici di Fisica fondamentale, PI: S. Degl'Innocenti) and by INFN (Iniziativa specifica TAsP).
Claret, A., and Torres, G. (2017). The dependence of convective core overshooting on stellar mass: a semi-empirical determination using the diffusive approach with two different element mixtures. Astrophys. J. 849:18. doi: 10.3847/1538-4357/aa8770

Clausen, J. V., Bruntt, H., Claret, A., Larsen, A., Andersen, J., Nordström, B., et al. (2009). Absolute dimensions of solar-type eclipsing binaries. II. V636 Centauri: A $1.05 M_{\odot}$ primary with an active, cool, oversize $0.85 M_{\odot}$ secondary. Astron. Astrophys. 502, 253-265. doi: 10.1051/0004-6361/200912362

Degl'Innocenti, S., Prada Moroni, P. G., Marconi, M., and Ruoppo, A. (2008). The FRANEC stellar evolutionary code. Astrophys. Space Sci. 316, 25-30. doi: 10.1007/s10509-007-9560-2

Deheuvels, S., and Michel, E. (2011). Constraints on the structure of the core of subgiants via mixed modes: the case of HD 49385. Astron. Astrophys. 535:A91. doi: 10.1051/0004-6361/201117232

Dell'Omodarme, M., Valle, G., Degl'Innocenti, S., and Prada Moroni, P. G. (2012). The pisa stellar evolution data base for low-mass stars. Astron. Astrophys. 540:A26. doi.org/10.1051/0004-6361/201118632 
Gallenne, A., Pietrzyski, G., Graczyk, D., Konorski, P., Kervella, P., Mrand, A., et al. (2016). The araucaria project: high-precision orbital parallax and masses of the eclipsing binary $\mathrm{tz}$ fornacis. Astron. Astrophys. 586:A35. doi: 10.1051/0004-6361/201526764

Gennaro, M., Prada Moroni, P. G., and Degl'Innocenti, S. (2010). $\Delta Y / \Delta Z$ from the analysis of local $\mathrm{K}$ dwarfs. Astron. Astrophys. 518:A13. doi: 10.1051/0004-6361/201014227

Guenther, D. B., and Demarque, P. (2000). $\alpha$ Centauri AB. Astrophys. J. 531, 503-520. doi: 10.1086/308446

Joyce, M., and Chaboyer, B. (2018a). Classically and asteroseismically constrained 1D stellar evolution models of $\alpha$ centauri A and B using empirical mixing length calibrations. Astrophys. J. 864:99. doi: 10.3847/1538-4357/aad464

Joyce, M., and Chaboyer, B. (2018b). Not all stars are the sun: empirical calibration of the mixing length for metal-poor stars using one-dimensional stellar evolution models. Astrophys. J. 856:10. doi: 10.3847/1538-4357/aab200

Krishna Swamy, K. S. (1966). Profiles of strong lines in K-dwarfs. Astrophys. J. 145:174.

Li, T., Bedding, T. R., Huber, D., Ball, W. H., Stello, D., Murphy, S. J., et al. (2018). Modelling Kepler red giants in eclipsing binaries: calibrating the mixinglength parameter with asteroseismology. Month. Notices R. Astronom. Soc. 475, 981-998. doi: 10.1093/mnras/stx3079

Ludwig, H.-G., Freytag, B., and Steffen, M. (1999). A calibration of the mixinglength for solar-type stars based on hydrodynamical simulations. I. Methodical aspects and results for solar metallicity. Astron. Astrophys. 346, 111-124.

Lydon, T. J., Fox, P. A., and Sofia, S. (1992). A formulation of convection for stellar structure and evolution calculations without the mixing-length theory approximations. I - application to the sun. Astrophys. J. 397, 701-716.

Magic, Z., Weiss, A., and Asplund, M. (2015). The Stagger-grid: a grid of 3D stellar atmosphere models. III. The relation to mixing length convection theory. Astron. Astrophys. 573:A89. doi: 10.1051/0004-6361/201423760

Mathur, S., Metcalfe, T. S., Woitaszek, M., Bruntt, H., Verner, G. A., Christensen-Dalsgaard, J., et al. (2012). A uniform asteroseismic analysis of 22 solar-type stars observed by kepler. Astrophys. J. 749:152. doi: 10.1088/0004-637X/749/2/152

Mosumgaard, J. R., Silva Aguirre, V., Weiss, A., Christensen-Dalsgaard, J., and Trampedach, R. (2017). "Improving 1D stellar models with 3D atmospheres," in European Physical Journal Web of Conferences, Vol 160, European Physical Journal Web of Conferences, 03009. doi: 10.1051/epjconf/201716003009

Peimbert, M., Luridiana, V., and Peimbert, A. (2007a). Revised primordial helium abundance based on new atomic data. Astrophys. J. 666, 636-646. doi: 10.1086/520571

Peimbert, M., Luridiana, V., Peimbert, A., and Carigi, L. (2007b). "On the primordial helium abundance and the $\Delta \mathrm{Y} / \Delta \mathrm{O}$ ratio," in From Stars to Galaxies: Building the Pieces to Build Up the Universe. Astronomical Society of the Pacific Conference Series, Vol. 374, eds A. Vallenari, R. Tantalo, L. Portinari, and A. Moretti (Venice: Istituto Veneto di Scienze, Lettere ed Arti), 81.

Stancliffe, R. J., Fossati, L., Passy, J.-C., and Schneider, F. R. N. (2015). Confronting uncertainties in stellar physics: calibrating convective overshooting with eclipsing binaries. Astron. Astrophys. 575:A117. doi: 10.1051/0004-6361/201425126

Tanner, J. D., Basu, S., and Demarque, P. (2013). Variation of stellar envelope convection and overshoot with metallicity. Astrophys. J. 767:78. doi: 10.1088/0004-637X/767/1/78

Thoul, A. A., Bahcall, J. N., and Loeb, A. (1994). Element diffusion in the solar interior. Astrophys. J. 421, 828-842.
Tognelli, E., Prada Moroni, P. G., and Degl'Innocenti, S. (2011). The Pisa pre-main sequence tracks and isochrones. A database covering a wide range of $\mathrm{Z}, \mathrm{Y}$, mass, and age values. Astron. Astrophys. 533:A109. doi: 10.1051/0004-6361/200913913

Trampedach, R., Stein, R. F., Christensen-Dalsgaard, J., Nordlund, A., and Asplund, M. (2014). Improvements to stellar structure models, based on a grid of 3D convection simulations - II. Calibrating the mixing-length formulation. Month. Notices R. Astronom. Soc. 445, 4366-4384. doi: 10.1093/mnras/ stu2084

Valle, G., Dell'Omodarme, M., Prada Moroni, P. G., and Degl'Innocenti, S. (2015a). Grid-based estimates of stellar ages in binary systems. SCEPtER: stellar charactEristics pisa estimation gRid. Astron. Astrophys. 579:A59. doi: 10.1051/0004-6361/201425568

Valle, G., Dell'Omodarme, M., Prada Moroni, P. G., and Degl'Innocenti, S. (2015b). Uncertainties in asteroseismic grid-based estimates of stellar ages. SCEPtER: Stellar CharactEristics Pisa Estimation gRid. Astron. Astrophys. 575:A12. doi: 10.1051/0004-6361/201424686

Valle, G., Dell'Omodarme, M., Prada Moroni, P. G., and Degl'Innocenti, S. (2016). A statistical test on the reliability of the non-coevality of stars in binary systems. Astron. Astrophys. 587:A31. doi: 10.1051/0004-6361/201526183

Valle, G., Dell'Omodarme, M., Prada Moroni, P. G., and Degl'Innocenti, S. (2017). Statistical errors and systematic biases in the calibration of the convective core overshooting with eclipsing binaries. A case study: TZ Fornacis. Astron. Astrophys. 600:A41. doi: 10.1051/0004-6361/201527336

Valle, G., Dell'Omodarme, M., Prada Moroni, P. G., and Degl'Innocenti, S. (2019). Mixing-length calibration from field stars. An investigation on statistical errors, systematic biases, and spurious metallicity trends. Astron. Astrophys. 623:A59. doi: 10.1051/0004-6361/201834949

Valle, G., Marconi, M., Degl'Innocenti, S., and Prada Moroni, P. G. (2009). Uncertainties on the theoretical predictions for classical Cepheid pulsational quantities. Astron. Astrophys. 507, 1541-1554. doi: 10.1051/0004-6361/200912004

Wu, X. S., Alexeeva, S., Mashonkina, L., Wang, L., Zhao, G., and Grupp, F. (2015). Calibrating the $\alpha$ parameter of convective efficiency using observed stellar properties. Astron. Astrophys. 577:A134. doi: 10.1051/0004-6361/ 201525781

Yildı, M. (2007). Models of $\alpha$ Centauri A and B with and without seismic constraints: time dependence of the mixing-length parameter. Month. Notices R. Astronom. Soc. 374, 1264-1270. doi: 10.1111/j.1365-2966.2006.11218.x

Yıldız, M., Yakut, K., Bakış, H., and Noels, A. (2006). Modelling the components of binaries in the Hyades: the dependence of the mixing-length parameter on stellar mass. Month. Notices R. Astronom. Soc. 368, 1941-1948. doi: 10.1111/j.1365-2966.2006.10275.x

Conflict of Interest Statement: The authors declare that the research was conducted in the absence of any commercial or financial relationships that could be construed as a potential conflict of interest.

Copyright (c) 2019 Valle, Dell'Omodarme, Prada Moroni and Degl'Innocenti. This is an open-access article distributed under the terms of the Creative Commons Attribution License (CC BY). The use, distribution or reproduction in other forums is permitted, provided the original author(s) and the copyright owner(s) are credited and that the original publication in this journal is cited, in accordance with accepted academic practice. No use, distribution or reproduction is permitted which does not comply with these terms. 